\title{
Contribution to the knowledge of the Aprostocetus Westwood, 1833 (Hymenoptera: Tetrastichinae) from Kırıkkale and Çankırı (Turkey) with some new records ${ }^{1}$
}

\author{
Kırıkkale ve Çankırı (Türkiye)'dan bazı yeni kayıtlar ile Aprostocetus Westwood, 1833 \\ (Hymenoptera: Tetrastichinae)'a ait bilgilere katkılar
}

Elif SAKALTAŞ ARIYAK ${ }^{2^{*}}$

\begin{abstract}
Summary
In this study species of genus Aprostocetus of Kırıkkale and Çankırı provinces are identified and data about some ecological aspects (locations, hosts, their distributions in the world) of the species are given. Samples were

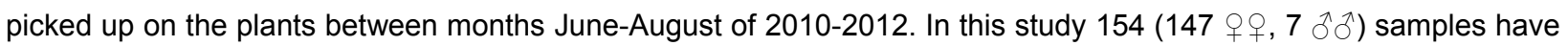
been evaluated. 29 species of genus Aprostocetus have been detected by identifying the samples from locations. 11 species of them are new records for the Turkish fauna. These species are; Aprostocetus aartseni Graham, 1987, A. artemisicola Graham, 1987, A. balasi (Erdös, 1954), A. constrictus Graham, 1987, A .flavifrons (Walker, 1849), A. incrassatus Graham, 1961, A. ligus (Walker, 1839), A. micantulus (Thomson, 1878), A. nubigenus Graham, 1986, A. palustris Graham, 1987, A. serratularum Graham, 1987. With this study the number of Aprostocetus species were updated to 60 species that was previously 49 species.
\end{abstract}

Key words: Tetrastichinae, Aprostocetus, Kırıkkale and Çankırı, Fauna, Turkey

\section{Özet}

Bu çalışmada Kırıkkale ve Çankırı'dan Aprostocetus cinsine ait türler tespit edilmiş ve türlerin bazı ekolojik özellikleri (toplama yapılan yerler, konukçuları, yayılışları) hakkında bilgiler verilmiştir. Örnekler 2010-2012 HaziranAğustos ayları arasında bitkiler üzerinden toplanmıştır. Bu çalışmada 154 (147 o+o, 7 ổ) örnek değerlendirilmiştir. Çalışma alanından toplanan örneklerin teşhislerinin yapılmasıyla Aprostocetus cinsine ait 29 tür tespit edilmiştir. Bunlardan 11 tür Türkiye için yeni kayıttır. Bu türler; Aprostocetus aartseni Graham, 1987, A. artemisicola Graham, 1987, A. balasi (Erdös, 1954), A. constrictus Graham, 1987, A. flavifrons (Walker, 1849), A. incrassatus Graham, 1961, A. ligus (Walker, 1839), A. micantulus (Thomson, 1878), A. nubigenus Graham, 1986, A. palustris Graham, 1987, A. serratularum Graham, 1987. Bu çalışmayla daha önce 49 olan Aprostocetus tür sayısı 60 olmuştur.

Anahtar sözcükler: Tetrastichinae, Aprostocetus, Kırıkkale ve Çankırı, Fauna, Türkiye

\footnotetext{
${ }^{1}$ This work is part of doctoral thesis submitted on 26 December 2013 in Ankara University Biology Department

${ }^{2}$ Yasemin Karakaya Science and Arts Center, 2432th blvd., 2489 street, Ümitköy/Ankara- Turkey

3 University of Ankara, Faculty of Science, Department of Biology 06100 Ankara-Turkey

"Sorumlu yazar (Corresponding author) e-mail: elifsakaltas@hotmail.com

Alını̧̧ (Received): 24.09.2014 Kabul ediliş (Accepted): 19.01.2015
} 


\section{Introduction}

Tetrastichinae is the most extensive subfamily of Eulophidae family of Hymenoptera (LaSalle, 1999). It is represented all over the world by over 1900 species in 110 genus and in Palearctic region 878 species in 48 genus (Noyes, 2014). Although most of it previously was grouped into Tetrastichus genus, this genus was divided into several natural groups later (Graham, 1987, 1991; Boucek, 1988). Hosts of this subfamily are grouped into 100 families of 10 different orders. Besides it has hosts that have features such as mites, spider eggs, phytophagous and even nematod (Graham, 1987; LaSalle, 1994; Sakaltaş \& Gençer, 2005). Some species of Tetrastichinae are used in biological control programs in various regions of the world. Some species of it are accepted as harmful since they attack to useful plants and insects. For this reason accurate diagnosis of Tetrastichinae used in biological control programs and found in ecosystems need to be conducted and also its biology and host need to be determined correctly. There are studies (Doğanlar, 1982, 1992a, 1992b, 1993a, 1993b, 1993c, 1993d, 1994, 2011; Gençer \& Doğanlar, 1995; Graham, 1987, 1991; Öncüer, 1991; Gençer, 2004; Sakaltaş \& Gençer, 2005; Çıkman \& Doğanlar, 2006; Doğanlar \& Doğanlar, 2008; Doğanlar et al., 2009; Gençer, 2009; Güler \& Kodon, 2010; Cebeci et al., 2011; Yegorenkova \& Yefremova, 2011; Koçak \& Özdemir, 2012) related to Tetrastichinae in our country. Fauna Tetrastichinae has reached 142 species in 16 genus in our country with the addition of this study (Sakaltaş, 2014).

Aprostocetus has the most species and is the most spread out genus of Tetrastichinae all over the World (Graham, 1987; Boucek, 1988; Noyes, 2014). Aprostocetus displays a wide range in host and biology. Many species of the subgenus Aprostocetus are primary parasitoids of hosts in plant galls, as Diptera Cecidomyiidae in particular, but also Hymenoptera, Cynipoidea, Coleoptera, Coccoidea, and eriophyid mites. Moreover, some species are occasional parasitoids of Diptera and Lepidoptera leafminers (Reina \& LaSalle, 2003). In various studies Aprostocetus Westwood, 1833 has been considered as a different genus besides it has been indicated that it was a synonym of Tetrastichus Haliday, 1844 by some authors (Doğanlar, 1992a). Recent studies provide synonyms, combinations, hosts, new records and new species of genus Aprostocetus (Doğanlar, 1992c, 1994, 2011; Gençer \& Doğanlar, 1995; Gençer, 2004; Sakaltaş \& Gençer, 2005; Yefremova \& Yegorenkova, 2005; Yefremova et al., 2007; Viggiani \& Monaco, 2014).

In this study 29 species of genus Aprostocetus of Kırıkkale and Çankırı provinces have been detected. 11 species of them are new records for the Turkish fauna. Detected species are written alphabetically and data about some ecological aspects of the species are given.

\section{Materials and Methods}

The materials for this study were collected by the first author from different locaties in Kırıkkale and Çankırı between June 2010-August 2012. Basically samples were collected by sweeping net on plants and mouth aspirator was used. Samples' localities were recorded by using GPS. Collected samples were places into glass tubes that have $70 \%$ ethyl alcohol in it. Diagnostic keys of Boucek (1988), Graham (1987), LaSalle (1994) were used for the diagnosis of the samples. Graham's diagnostic key that was developed for European fauna was used mostly among those diagnostic keys. Diagnostic keys have been formed by revising this diagnostic key. Leica S8 APO stereozoom microscope has been used to diagnose samples. Species diagnosis keys have been formed after species have been diagnosed and preparations have been formed in order to show characters, lastly their pictures were taken by Nikon E 4500 digital camera.

Spread out of the species and their hosts identifications were made by using diagnostic keys and other related literature (Doğanlar, 1994; Gençer \& Doğanlar, 1995; Gençer, 2004; Sakaltaş \& Gençer, 2005; Doğanlar, 2011; Noyes, 2014). Samples are preserved in Entomology Museum of Ankara University Biology department. 


\section{Results}

In this study 29 species of genus Aprostocetus Westwood, 1833 (Hymenoptera: Eulophidae: Tetrastichinae) of Kırıkkale and Çankırı provinces have been identified. 11 species of them are new records for fauna of the Turkish fauna. Some ecological aspects like distribution in the World, hosts of the species detected in working fields are given.

Aprostocetus aartseni Graham, 1987

Material Examined: Turkey, Çankırı: Akçakavak village, 24.06.2012, 1우 Merkez, 713 m, N:40 58'24"/E:3362'10", 24.VI.2012, 19, (Leg. Sakaltaş Arıyak).

Host: Unknown.

Distribution: Greece (Graham, 1987; Noyes, 2014).

New record for Turkey.

Aprostocetus annulatus (Förster, 1861)

Material Examined: Turkey, Çankırı: Atkaracalar, $1237 \mathrm{~m}, \mathrm{~N}: 40^{\circ} 81^{\prime} 16 " / \mathrm{E}: 33^{\circ} 07^{\prime} 35^{\prime \prime}, 23 . \mathrm{VII} .2011$,

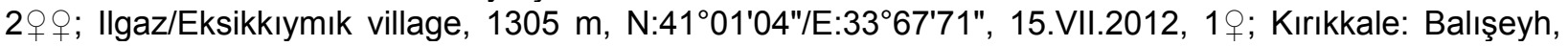
$865 \mathrm{~m}, \mathrm{~N}: 39^{\circ} 90^{\prime} 91^{\prime \prime} / \mathrm{E}: 33^{\circ} 71^{\prime} 15^{\prime \prime}$, 12.VII.2010, 1우 Bahşlı/Celal Bayar Park, 686 m, 08.VII. 2012, $\mathrm{N}: 39^{\circ} 81^{\prime} 72 " / \mathrm{E}: 33^{\circ} 48^{\prime} 06^{\prime \prime}, 1$ \% , (Leg. Sakaltaş Arıyak).

Host: Asphondylia sarothamni (Diptera: Cecidomyiidae), Eulecanium corni, Sphaerolecanium prunastri (Hemiptera: Coccidae) (Noyes, 2014).

Distribution: France, Germany, Hungary, Russia, Sweden, Switzerland, Ukraine, England (Noyes, 2014), Turkey; Sivas: Campus (Gençer, 2004).

Aprostocetus artemisicola Graham, 1987

Material Examined: Turkey, Çankırı: Ilgaz/Kırkpınar plateau, 1794 m, N:4100'49"/E:3361'85", 15.VII.2012, 1; Ilgaz/Ödemiş village, 969 m, N:4093'74"/E:3356'97", 15.VII.2012, 1+; Kırıkkale: Bahşılı/Celal Bayar Park, 686 m, N:3981'72"/E:3348'06", 08.VII.2012, 1o; Keskin, 1056 m, N:39 70'69"/E:3357'91", 13.VII.2010, 1ㅇ, (Leg. Sakaltaş Arıyak).

Host: Contarinia artemisiae (Diptera: Cecidomyiidae), Artemisia sp., A. arborescens, A. vulgaris (Asteraceae) (Noyes, 2014).

Distribution: Germany, Italy, Russia, Spain, Sweden, England (Noyes, 2014).

New record for Turkey.

Aprostocetus bakkendorfi Graham, 1987

Material Examined: Turkey, Çankırı: Hasakçe village, 845 m, N:4065'87"/E:3376'62", 23.VI. 2012, 1, (Leg. Sakaltaş Arıyak).

Distribution: Denmark, Russia, Sweden (Noyes, 2014), Turkey; Tokat (Doğanlar, 1994).

Host: Cecidomyiidae, Astragalus glycyphyllos (Fabaceae) (Noyes, 2014).

Aprostocetus balasi (Erdös, 1954)

Material Examined: Turkey, Kırıkkale: Balışeyh, 865 m, N:3990'91"/E:3371'15", 12.VII.2010, 4 우, (Leg. Sakaltaş Arıyak).

Host: Phyllocnistis suffusella, Phyllocnistis unipunctella (Lepidoptera: Gracillariidae), Pseudargyrotoza conwagana (Lepidoptera: Tortricidae), Fraxinus sp. (Oleaceae) (Noyes, 2014).

Distribution: Austria, France, Hungary, Italy, Sweden (Noyes, 2014).

New record for Turkey. 
Aprostocetus catius (Walker, 1839)

Material Examined: Turkey, Çankırı: Hasakçe village, 845 m, N:4065'87"/E:3376'62", 23.VI. 2012,

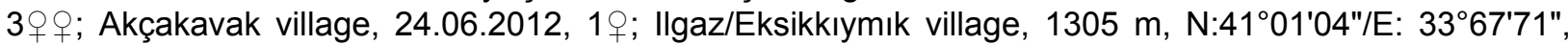
15.VII.2012, 4우; Ilgaz/Ödemiş village, $969 \mathrm{~m}, \mathrm{~N}: 40^{\circ} 93^{\prime} 74 " / \mathrm{E}: 33^{\circ} 56^{\prime} 97 "$ ", 15.VII.2012, 1; Kırıkkale: Çelebi, 1093 m, N:3945'31"/E:3355'03", 13.VII.2010, 1; Keskin, 1056 m, N:3970'69"/E: 3357'91",

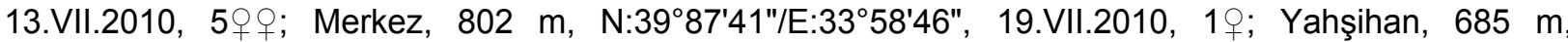
19.VII.2010, N:3984'03"/E:3345'51", 1 \% , (Leg. Sakaltaş Arıyak).

Host: In some types graminae Cecidomyiidae (Diptera) was collected over (Doğanlar, 1994).

Distribution: Czech Republic, Czechoslovakia, Ireland (North and South), Russia, Sweden, Turkey, England (Noyes, 2014), Turkey; Tokat (Doğanlar, 1994), Ankara: Altındağ/Altınova, Elmadağ: Hasanoğlan, Haymana, Güdül, Söğütözü (Sakaltaş \& Gençer, 2005), Sivas: Campus, Kırşehir: Mucur (Gençer, 2004).

\section{Aprostocetus cerricola (Erdös, 1954)}

Material Examined: Turkey, Çankırı: Merkez, 713 m, N:4058'24"/E:3362'10", 24.VI.2012, 4우, (Leg. Sakaltaş Arıyak).

Host: Macrodiplosis dryobia (Diptera: Cecidomyiidae), Andricus grossulariae (Hymenoptera: Cynipidae), Arnoldiola cerris (Diptera: Cecidomyiidae), Quercus cerris (Fagaceae) (Noyes, 2014).

Distribution: Austria, Belgium, Czechoslovakia, France, Greece, Hungary, Russia, Slovakia, Spain, Sweden, Yugoslavia (pre 1991) (Noyes, 2014), Turkey; Konya: Ereğli, Tokat: Almus (Gençer, 2004).

Aprostocetus crino (Walker, 1838)

Material Examined: Turkey, Çankırı: Eldivan, 915 m, N:4054'21"/E:3350'61", 23.VII.2011, 6o $ᄋ$; Merkez, 713 m, N:4058'24"/E:3362'10", 24.VI.2012, 1+; Şabanözü, 1109 m, N:4047'78"/E: 33³0'02", 23.VII.2011, 2o+ ; Kırıkkale: Çelebi, 1093 m, N:3945'31"/E:3355'03", 13.VII.2010, 1q, (Leg. Sakaltaş Arlyak).

Host: Yponomeuta padellus (Lepidoptera: Yponomeutidae), Oecanthus sp., O. nigricornis, O. pellucens, O. quadripunctatus (Orthoptera: Gryllidae), Padus racemosa (Rosaceae) (Noyes, 2014).

Distribution: Andorra, Bulgaria, Croatia, Czech Republic, Czechoslovakia, Denmark, France, Germany, Hungary, Iceland, Ireland (North and South), Italy, Moldova, Nearctic, Netherlands, Peoples' Republic of China, Russia, Slovakia, Sweden, Turkey; Ankara: Altındağ/Altınova (Sakaltaş \& Gençer, 2005), England, USA, Yugoslavia (Federal Republic), Yugoslavia (pre 1991) (Noyes, 2014).

Aprostocetus constrictus Graham, 1987

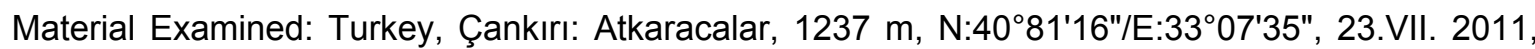
1; Merkez, $713 \mathrm{~m}, 24.06 .2012, \mathrm{~N}: 40^{\circ} 58^{\prime} 24^{\prime \prime} / \mathrm{E}: 33^{\circ} 62^{\prime} 10^{\prime \prime}, 4$ 우우 Ilgaz/Eksikkıymık village, $1305 \mathrm{~m}$,

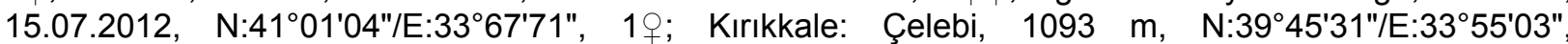
13.07.2010, 1 \%; Keskin, 1056 m, N:3970'69"/E:3357'91", 13.VII.2010, 6우, (Leg. Sakaltaş Arıyak).

Host: Apion sp. (Coleoptera: Apionidae), Oligotrophus sp., O. skuhravae, O. tarda (Diptera: Cecidomyiidae), Betula pontaninii, B. pubescens (Betulaceae) (Noyes, 2014).

Distribution: Netherlands, Norway, Russia, Sweden, England (Noyes, 2014).

New record for Turkey.

\section{Aprostocetus emesa (Walker, 1839)}

Material Examined: Turkey, Çankırı: Hasakçe village, 845 m, N:4065'87"/E:3376'62", 23.VI. 2012, 3우; Kırıkkale: Bahşılı/Celal Bayar Park, 697 m, N:3981'57"/E:3347'71", 08.VII.2010, 1q, (Leg. Sakaltaş Arıyak). 
Host: Dasineura alopecuri (Diptera: Cecidomyiidae) (Noyes, 2014).

Distribution: Czech Republic, Czechoslovakia, France, Germany, Hungary, Ireland (North and South), Netherlands, Norway, Russia, Slovakia, Sweden, England (Noyes, 2014), Turkey; Tokat (Doğanlar, 1994), Sivas: Yıldızeli, Yozgat-Kayseri main road, Düver (Gençer, 2004).

Aprostocetus flavifrons (Walker, 1849)

Material Examined: Turkey, Kırıkkale: Balışeyh, 865 m, N:3990'91"/E:3371'15", 12.VII.2010, 1ㅇ, (Leg. Sakaltaş Arıyak). 2014).

Host: Cerodontha pygmaea (Diptera: Agromyzidae), Brachypodium sylvaticum (Poaceae) (Noyes,

Distribution: Italy, Madeira (Noyes, 2014).

New record for Turkey.

Aprostocetus forsteri (Walker, 1847)

Material Examined: Turkey, Kırıkkale: Balışeyh, 865 m, N:3990'91"/E:3371'15", 12.VII.2010, 1 ; ; Keskin, 1056 m, N:3970'69"/E:3357'91", 13.VII.2010, 2우, (Leg. Sakaltaş Arıyak).

Distribution: Austria, Bulgaria, Cyprus, Czech Republic, Czechoslovakia, France, Hungary, Iran, Italy, Palaearctic, Russia, Slovakia, Spain, Sweden, Switzerland (Noyes, 2014), Turkey; Tokat: Artova, Gürcüköy, Erzurum, Adana: Belemedik, Karaisalı (Doğanlar, 1992a), Sivas: Hara, Campus, Paşabahçe, Türk-iş Blocks (Gençer \& Doğanlar, 1995), Ankara: Ayaş, Beypazarı, Çankaya/Beytepe village, Çayırhan, Güdül, Haymana, Sincan (Sakaltaş \& Gençer, 2005).

Host: Tephritis neesii (Diptera: Tephritidae), Aulax rogenhoferi, A. salviae, Aylax jaceae, A. rogenhoferi, A. salviae, Barbotinia oraniensis, Diplolepis mayri, Isocolus jaceae, I. rogenhoferi, I. scabiosae (Hymenoptera: Cynipidae), Urophora sp. (Diptera: Tephritidae), Centaurea jacea, C. nigra, C. paniculata, C. pannonica, C. sadleriana, C. scabiosa, Leucanthemum vulgare (Asteraceae), Salvia lavandulifolia, S. nemorosa, S. officinalis, S. pratensis (Lamiaceae), Papaver dubium, P. rhoeas (Papaveraceae), Rosa sp. (Rosaceae) (Noyes, 2014).

Aprostocetus fusificola Graham, 1987

Material Examined: Turkey, Çankırı: Hasakçe village, 845 m, N:4065'87"/E:3376'62", 23.VI. 2012, 19; Ilgaz/Eksikkıymık village, $1305 \mathrm{~m}, \mathrm{~N}: 41^{\circ} 01^{\prime} 04^{\prime \prime} / \mathrm{E}: 33^{\circ} 67^{\prime} 71 ", 15 . \mathrm{VII.2012,} 1$ \%; Merkez, $713 \mathrm{~m}$,

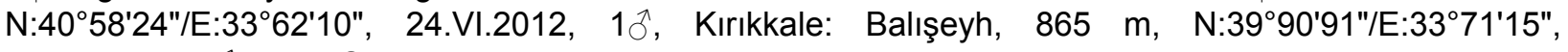
12.VII.2010, 1 \%, (Leg. Sakaltaş Arıyak).

Host: Plagiotrochus cardiguensis, P. fusifex, P. quercusilicis (Hymenoptera: Cynipidae), Quercus coccifera, Q. ilex (Fagaceae) (Noyes, 2014).

Distribution: France, Spain (Noyes, 2014), Turkey; Yozgat, Düver (Gençer, 2004).

Aprostocetus incrassatus Graham, 1961

Material Examined: Turkey, Çankırı: Merkez, 713 m, N:4058'24"/E:3362'10", 24.VI.2012, 19, (Leg. Sakaltaş Arıyak).

Host: Carex sp. (Cyperaceae) (Noyes, 2014).

Distribution: Russia, Sweden, England (Noyes, 2014).

New record for Turkey. 
Aprostocetus larzacensis Graham, 1987

Material Examined: Turkey, Çankırl: Hasakçe village, 845 m, N:4065'87"/E:3376'62", 23.VI. 2012, 1; Ilgaz/Eksikkıymık village, $1305 \mathrm{~m}, \mathrm{~N}: 41^{\circ} 01^{\prime} 04^{\prime \prime} / \mathrm{E}: 33^{\circ} 67^{\prime} 71 "$, 15.VII.2012, 5우, (Leg. Sakaltaş Arıyak).

Host: Unknown.

Distribution: France, Moldova, Russia, Sweden (Noyes, 2014), Turkey; Tokat (Doğanlar, 1994).

Aprostocetus levadiensis Graham, 1987

Material Examined: Turkey, Kırıkkale: Delice/Alcılı village, 639 m, N:3999'73"/E:3406'45", 12. VII.2010, 19, (Leg. Sakaltaş Arıyak).

Host: Unknown.

Distribution: Greece, Sweden (Noyes, 2014), Turkey; Tokat (Doğanlar, 1994), Ankara: Bağlum (Sakaltaş \& Gençer, 2005), Sivas: Campus, Kırşehir: Hirfanlı Dam (Gençer, 2004).

Aprostocetus ligus (Walker, 1839)

Material Examined: Turkey, Çankırı: Eldivan, 965 m, N:4052'31"/E:3349'80", 23.VI.2012, 3우, (Leg. Sakaltaş Arıyak).

Host: Unknown.

Distribution: Russia, Sweden, England (Noyes, 2014).

New record for Turkey.

Aprostocetus micantulus (Thomson, 1878)

Material Examined: Turkey, Çankırı: Merkez, 713 m, N:4058'24"/E:3362'10", 24.VI.2012, 6우; Kırıkkale: Balışeyh, 865 m, N:3990'91"/E:3371'15", 12.VII.2010, 1ㅇ, (Leg. Sakaltaş Arıyak).

Host: Dasineura abietiperda (Diptera: Cecidomyiidae), Pinus sp. (Pinaceae) (Noyes, 2014).

Distribution: France, Hungary, İtaly, Russia, Sweden, United Kingdom (Noyes, 2014).

New record for Turkey.

Aprostocetus nubigenus Graham, 1986

Material Examined: Turkey, Çankırı: Merkez, 713 m, N:4058'24"/E:3362'10", 24.VI.2012, 2o+ (Leg. Sakaltaş Arıyak).

Host: Unknown.

Distribution: Madeira, Peoples' Republic of China (Noyes, 2014).

New record for Turkey.

Aprostocetus palustris Graham, 1987

Material Examined: Turkey, Çankırı: Merkez, 713 m, N:4058'24"/E:3362'10", 24.VI.2012, 19, Kırıkkale: Balışeyh, 865 m, N:3990'91"/E:3371'15", 12.VII.2010, 19; Bahşılı/Karaahmetli Dam, 718 m, $\mathrm{N}: 39^{\circ} 66^{\prime} 64^{\prime \prime} / \mathrm{E}: 33^{\circ} 44^{\prime} 47^{\prime \prime}, 14 . \mathrm{VII} .2010,1$, , (Leg. Sakaltaş Arıyak).

Host: Unknown.

Distribution: Sweden, England (Noyes, 2014).

New record for Turkey. 
Aprostocetus pausiris (Walker, 1839)

Material Examined: Turkey, Çankırl: Atkaracalar, 1237 m, N:4081'16"/E:3307'35, 23.VII. 2011, 13; Eldivan, $915 \mathrm{~m}, \mathrm{~N}: 40^{\circ} 54^{\prime} 21^{\prime \prime} / \mathrm{E}: 33^{\circ} 50^{\prime} 61 ", 23 . V I I .2011,1$; ; Ilgaz/Eksikkıymık village, $1305 \mathrm{~m}$, $\mathrm{N}: 41^{\circ} 01^{\prime} 04^{\prime \prime} / \mathrm{E}: 33^{\circ} 67^{\prime} 71 ", 15 . \mathrm{VII.2012,} 1$ \%; Ilgaz/Kırkpınar plateau, $1794 \mathrm{~m}, \mathrm{~N}: 41^{\circ} 00^{\prime} 49^{\prime \prime} / \mathrm{E}: 33^{\circ}$ 61' 85",

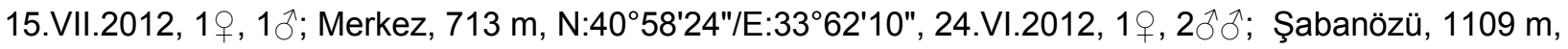

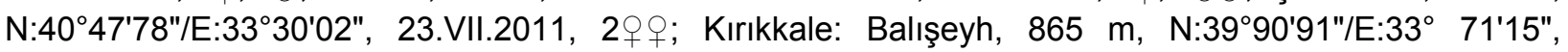
12.VII.2010, 5우으, (Leg. Sakaltaş Arıyak).

Host: Dasineura leguminicola (Diptera: Cecidomyiidae), Lipara sp. (Diptera: Chloropidae), Phragmites australis (Poaceae) (Noyes, 2014).

Distribution: Canada, Czech Republic, Czechoslovakia, Denmark, France, Germany, Greece, Hungary, Ireland (North and South), Madeira, Nearctic, Netherlands, Norway, Portugal, Russia, Spain, Sweden, Turkey; Tokat (Doğanlar, 1994), Ankara: Akyurt, Altınova, Bağlum, Batıkent, Çubuk Highways Park, Söğütözü (Sakaltaş \& Gençer, 2005), Sivas: Campus, Kırşehir-Kayseri mainroad 130 km (Gençer, 2004) England, USSR, Yugoslavia (pre 1991) (Noyes, 2014).

Aprostocetus phineus (Walker, 1839)

Material Examined: Turkey, Çankırı: Eldivan, 915 m, N:4054'21"/E:3350'61", 23.VII.2011, 19; Eldivan, $965 \mathrm{~m}, \mathrm{~N}: 40^{\circ} 52^{\prime} 31^{\prime \prime} / \mathrm{E}: 33^{\circ} 49^{\prime} 80 ", 23 . V I .2012,3$ \% $q$; Hasakçe village, $845 \mathrm{~m}, \mathrm{~N}: 40^{\circ} 65^{\prime} 87^{\prime \prime} / \mathrm{E}$ :

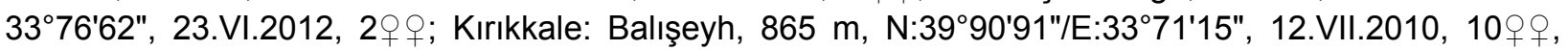
(Leg. Sakaltaş Arıyak).

Host: These species generally occur in grassy habitats (Gençer \& Doğanlar, 1995).

Distribution: Czech Republic, Czechoslovakia, Denmark, France, Germany, Hungary, Slovakia, Sweden, England, USSR, Yugoslavia (pre 1991), Turkey; Sivas: Campus, Hara, Paşabahçe (Gençer \& Doğanlar, 1995).

Aprostocetus pygmaeus (Zetterstedt, 1838)

Material Examined: Turkey, Çankırı: Eldivan, 915 m, N:4054'21"/E:3350'61", 23.VII.2011, 19;

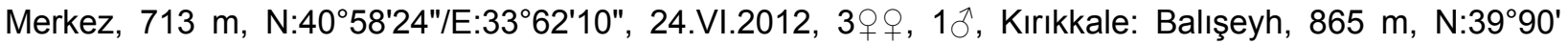

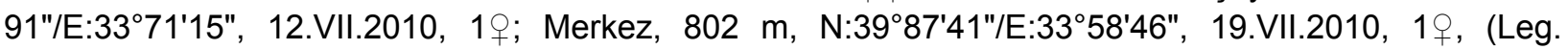
Sakaltaş Arıyak).

Host: Cecidomyiidae unspecified, Dasineura alopecuri (Diptera: Cecidomyiidae), Megachile centuncularis, Osmia coerulescens (Hymenoptera: Apidae), Diplolepis rosae (Hymenoptera: Cynipidae), Alopecurus pratensis (Poaceae), Rosa sp. (Rosaceae) (Noyes, 2014).

Distribution: Canada, Czech Republic, Czechoslovakia, Denmark, Europe, Finland, France, Germany, Greece, Hungary, Italy, Netherlands, Norway, Russia, Sweden, Turkey; Kars: Ardahan, Aralık, Tokat: Fidanlık, Taşçiftlik, Zile, Pazar (Doğanlar, 2004; Gençer \& Doğanlar, 1995), Ankara; Çamlıdere, Lalahan, Ayaş, Şereflikoçhisar, Mamak/Kayaş, Hasanoğlan (Sakaltaş \& Gençer, 2005), Ukraine, England (Noyes, 2014).

Aprostocetus productus Graham, 1987

Material Examined: Turkey, Çankırı: Ilgaz/Eksikkıymık village, 1305 m, N:4101'04"/E:3367' 71", 15.VII.2012, 4우; Kırıkkale: Keskin, 1056 m, N:3970'69"/E:3357'91", 13.VII.2010, 1ㅇ, (Leg. Sakaltaş Arlyak).

Host: Unknown. 
Distribution: France, Russia, Sweden (Noyes, 2014), Turkey; Tokat (Doğanlar, 1994), Sivas: Paşabahçe, Campus, Tuzlugöl (Gençer \& Doğanlar, 1995).

\section{Aprostocetus serratularum Graham, 1987}

Material Examined: Turkey, Kırıkkale: Balışeyh, 865 m, 12.VII.2010, N:3990'91"/E:3371'15", 1 q, (Leg. Sakaltaş Arıyak).

Host: Terellia serratulae, Urophora cuspidata, U. jaceana, U. solstitialis (Diptera: Tephritidae), Metzneria sp. (Lepidoptera: Gelechiidae), Carduus nutans, Centaurea lanceolatum, C. nigra, C. scabiosa, Cirsium sp., (Asteraceae) (Noyes, 2014).

Distribution: Czech Republic, Germany, Greece, Italy, Netherlands, Russia, Sweden, England (Noyes, 2014).

New record for Turkey.

Aprostocetus tymber (Walker, 1839)

Material Examined: Turkey, Çankırı: Şabanözü, 1109 m, N:4047'78"/E:33³0'02", 23.VII.2011, 2 우오, (Leg. Sakaltaş Arıyak).

Host: Helicomyia saliciperda, Lasioptera rubi (Diptera: Cecidomyiidae), Rubus sp., R. idaeus (Rubiaceae), Salix sp. (Salicaceae) (Noyes, 2014).

Distribution: Czech Republic, France, Ireland (North and South), Italy, Netherlands, Poland, Russia, Slovakia, Sweden, Turkey; Ankara: Söğütözü (Sakaltaş \& Gençer, 2005), England, Yugoslavia (Federel Republic), Yugoslavia (pre 1991) (Noyes, 2014).

Aprostocetus toddaliae (Risbec, 1958)

Material Examined: Turkey, Çankırı: Ilgaz/Eksikkıymık Village, 1305 m, N:4101'04"/E:3367' 71", 15.VII.2012, 1; Ilgaz/Ödemiş village, 969 m, N:4093'74"/E:3356'97", 15.VII.2012, 4우; Kırıkkale: Balışeyh, 865 m, N:3990'91"/E:3371'15", 12.VII.2010, 1q, (Leg. Sakaltaş Arıyak).

Host: Ceroplastes sp. (Hemiptera: Coccidae) (Noyes, 2014), C. rusci L., Citrus paradisi Matc., Citrus sinensis Del Guer, Ficus carica (Tetrastichus ceroplastae) (Doğanlar, 1994).

Distribution: Israel, Madagascar, Turkey; Antalya, İzmir, Muğla (Doğanlar, 1994; Noyes, 2014).

Aprostocetus venustus (Gahan, 1914)

Material Examined: Turkey, Çankırı: Akçakavak Village, 24.VI.2012, 1\%; Merkez, 713 m, N:40 58'24"/E:3362'10", 24.VI.2012, 19; Kırıkkale: Keskin, 1056 m, N:3970'69"/E:3357'91", 13.VII.2010, $2 ㅇ ㅜ$, (Leg. Sakaltaş Arıyak).

Host: Asphondylia websteri, Contarinia sorghicola (Diptera: Cecidomyiidae), Aulacidea freesei (Hymenoptera: Cynipidae), Bruchophagus sp., B. gibbus, B. onobrychidis, B. roddi (Hymenoptera: Eurytomidae), Notobasis syriaca, Silybum marianum (Asteraceae), Medicago sativa, Onobrychis sp. (Fabaceae), Sorghum sp. (Poaceae) (Noyes, 2014).

Distribution: Bulgaria, Canada, Canary Islands, Czech Republic, Czechoslovakia, Europe, France, Germany, Hungary, Iran, Italy, Nearctic, Netherlands, Palaearctic, Russia, Slovakia, Spain, Sweden, USA, USSR, Yugoslavia (pre 1991) (Noyes, 2014), Turkey; Erzurum (Doğanlar, 1994). 
Aprostocetus viridinitens Graham, 1987

Material Examined: Turkey, Çankırı: Merkez, 713 m, N:4058'24"/E:3362'10", 24.VI.2012, 1 \%; Kırıkkale: Balışeyh, 865 m, N:3990'91"/E:3371'15", 12.VII.2010, 2우, (Leg. Sakaltaş Arıyak).

Host: Artemisia vulgaris, collected on Dolden and Betula (Doğanlar, 1994).

Distribution: Czech Republic, France, Russia, Sweden, England (Noyes, 2014), Turkey; Tokat: Taşlıçiftlik, Fidanlık, Turhal, Şenyurt, Ö. Sunar Farm (Doğanlar, 1992a; Doğanlar, 1994).

\section{Conclusion}

Turkey, a rich country in the sense of biological variety, is a bridge country at the intersection area of Asia, Europe and Africa continents and also has fauna and flora elements of these three continents because of its different climate conditions (Gülperçin \& Tezcan, 2010).

Kırıkkale and Çankırı located in the middle of Central Anatolia, are at the intersection points between east-west, north-south regarding their topographical structures, climatic conditions, vegetations, temperatures etc. For this reason, effects of climatic condition and vegetation of Black Sea can be seen in most parts of the regions in addition to their own terrestrial climate. This situation increases their flora and fauna richness. Fauna Aprostocetus of Kırıkkale and Çankırı has been identified with this study. 11 new records have been identified for Turkey [Aprostocetus aartseni Graham, 1987, A.artemisicola Graham, 1987, A. balasi (Erdös, 1954), A. constrictus Graham, 1987, A. flavifrons (Walker, 1849), A. incrassatus Graham, 1961, A. ligus (Walker, 1839), A. micantulus (Thomson, 1878), A. nubigenus Graham, 1986, $A$. palustris Graham, 1987, A. serratularum Graham, 1987] and Turkish Aprostocetus species number has become 60. Turkish Aprostocetus species number was 49 prior to this study (Table 1). There are 704 species in the World and 324 species in Palearctic of this genus (Noyes, 2014). Turkish Aprostocetus species are $8.5 \%$ of total Aprostocetus species in the World and $18.5 \%$ of Aprostocetus species in Palearctic considering the new records in this study. These ratios are relatively low compared to the World and Palearctic regions. More faunistical studies must be conducted in order to discover the richness of Turkish Aprostocetus fauna.

Aprostocetus are parasitic or hiperparasitic in Lepidoptera (Yponomeutidae, Gelechiidae, Gracillariidae, Tortricidae), Orthoptera (Gryllidae), Diptera (Cecidomyiidae, Chloropidae, Tephritidae), Hymenoptera (Cynipidae, Eurytomidae, Apidae), Coleoptera (Apionidae) and Hemiptera (Coccidae). As a result, diagnoses of samples pertaining to Aprostocetus species which are used in biological control programs and also exist in ecosystems need to be done very well and to be determined their biologies and hosts.

Aim of this study is to contribute to the fauna Tetrastichinae in Turkey and help researchers who are to apply biological control program using ecological observations related to the samples' natural habitats and their pick up time. 
Contribution to the knowledge of the Aprostocetus Westwood, 1833 (Hymenoptera: Tetrastichinae ) from Kırıkkale and Çankırı (Turkey) with some new records

Table 1. Previously recorded Aprostocetus species and literatures in Turkey

\begin{tabular}{|c|c|}
\hline Species Name & Literature \\
\hline Aprostocetus agevilleae Graham, 1987 & Gençer, 2004; Sakaltaş \& Gençer, 2005 \\
\hline Aprostocetus agrus (Walker, 1839) & Doğanlar, 1994; Sakaltaş \& Gençer, 2005 \\
\hline Aprostocetus annulatus (Förster, 1861) & Gençer, 2004 \\
\hline Aprostocetus arenarius (Erdös, 1954) & Doğanlar, 1994 \\
\hline Aprostocetus artemisiae (Erdös, 1954) & Doğanlar, 1994 \\
\hline Aprostocetus bakkendorfi Graham, 1987 & Doğanlar, 1994; Sakaltaş, 2014 \\
\hline Aprostocetus brachycerus (Thomson, 1878) & Doğanlar, 1994 \\
\hline Aprostocetus bruzzonis (Masi, 1930) & Doğanlar, 1994 \\
\hline Aprostocetus bucculentus (Kostjukov, 1978) & Graham, 1987; Doğanlar, 1994 \\
\hline Aprostocetus catius (Walker, 1839) & Doğanlar, 1994; Gençer, 2004; Sakaltaş \& Gençer, 2005; Sakaltaş, 2014 \\
\hline Aprostocetus caudatus Westwood, 1833 & Sakaltaş \& Gençer, 2005 \\
\hline Aprostocetus ceroplastae (Girault, 1916 ) & $\begin{array}{l}\text { Domenichini, 1966; Graham, 1987, OILB, 1971; Öncüer, 1991; Özsemerci \& } \\
\text { Akşit, 2003, Prinsloo, } 1984 .\end{array}$ \\
\hline Aprostocetus cerricola (Erdös, 1954) & Gençer, 2004; Sakaltaş \& Gençer, 2005; Sakaltaş, 2014 \\
\hline Aprostocetus citrinus (Förster, 1841 & Gençer, 2004 \\
\hline Aprostocetus coccidiphagus Graham, 1987 & Gençer, 2004 \\
\hline Aprostocetus cracens Graham, 1987 & Kaya \& Kovancı, 2001 \\
\hline Aprostocetus emesa (Walker, 1839) & Doğanlar, 1994; Gençer, 2004; Sakaltaş, 2014 \\
\hline Aprostocetus epicharmus (Walker, 1839) & Graham, 1987; Doğanlar, 1994 \\
\hline Aprostocetus eriophyes (Taylor, 1909) & Öncüer, 1991 \\
\hline Aprostocetus forsteri (Walker, 1847) & Doğanlar, 1994; Sakaltaş \& Gençer, 2005; Sakaltaş, 2014 \\
\hline Aprostocetus fusificola Graham, 1987 & Gençer, 2004; Sakaltaş, 2014 \\
\hline Aprostocetus gaus (Walker, 1839) & Gençer, 2004 \\
\hline Aprostocetus grandii (Domenichini, 1965) & Gençer, 2004 \\
\hline Aprostocetus grylli (Erdös, 1954) & Gençer, 2004 \\
\hline Aprostocetus holoxanthus Graham, 1987 & Graham, 1987 \\
\hline Aprostocetus larzacensis Graham, 1987 & Doğanlar, 1994; Sakaltaş, 2014 \\
\hline Aprostocetus lasiopterus Doganlar, 2011 & Doğanlar, 2011 \\
\hline Aprostocetus levadiensis Graham, 1987 & Doğanlar, 1994; Sakaltaş \& Gençer, 2005; Sakaltaş, 2014 \\
\hline Aprostocetus longicauda (Thomson, 1878) & Graham, 1987; Doğanlar, 1994 \\
\hline Aprostocetus lycidas (Walker, 1839 ) & OILB, 1971 \\
\hline Aprostocetus mimulus Graham, 1987 & Gençer, 2004 \\
\hline Aprostocetus neglectus (Domenichini, 1957) & Graham, 1987; Öncüer, 1991; Doğanlar, 1994 \\
\hline Aprostocetus pausiris (Walker, 1839) & Doğanlar, 1994; Gençer, 2004, Sakaltaş \& Gençer, 2005; Sakaltaş, 2014 \\
\hline Aprostocetus phineus (Walker, 1839) & Gencer \& Doğanlar, 1995; Gençer, 2004; Sakaltas, 2014 \\
\hline Aprostocetus phragmiticola Graham, 1987 & Gençer, 2004 \\
\hline Aprostocetus productus Graham, 1987 & Doğanlar, 1994; Sakaltaş \& Gençer, 2005; Sakaltaş, 2014 \\
\hline Aprostocetus pygmaeus (Zetterstedt, 1838) & Doğanlar, 1994; Sakaltaş \& Gençer, 2005; Sakaltaş, 2014 \\
\hline Aprostocetus rumicis Graham, 1987 & Doğanlar, 1994 \\
\hline Aprostocetus samandagus Doganlar, 2011 & Doğanlar, 2011 \\
\hline Aprostocetus subcylindricus Graham, 1987 & Doğanlar, 1994 \\
\hline Aprostocetus tenuiradialis Graham, 1987 & Doğanlar, 1994 \\
\hline Aprostocetus terebrans Erdös, 1954 & Sakaltaş \& Gençer, 2005 \\
\hline Aprostocetus toddaliae (Risbec, 1958) & Graham, 1987; Doğanlar, 1994 \\
\hline Aprostocetus tompanus (Erdös, 1954) & Doğanlar, 1994; Gençer, 2004 \\
\hline Aprostocetus trjapitzini (Kostjukov, 1976) & Ülgentürk, 2001; Karaca, et al., 2003; Ülgentürk \& Toros, 2001 \\
\hline Aprostocetus tymber (Walker, 1839) & Sakaltas \& Gençer, 2005; Sakaltaş, 2014 \\
\hline Aprostocetus venustus (Gahan, 1914) & Doğanlar, 1994; Sakaltaş, 2014 \\
\hline Aprostocetus viridinitens Graham, 1987 & Doğanlar, 1994; Sakaltaş, 2014 \\
\hline Aprostocetus westwoodii (Fonscolombe, 1840) & Doğanlar, 1994 \\
\hline
\end{tabular}

\section{Acknowledgements}

This work is part of doctoral thesis submitted on 26 December 2013 in Ankara University Biology Department. I would like to thank Prof. Dr. Mikdat DOĞANLAR for his significant contributions for this study and also my family for their support. 


\section{References}

Boucek, Z., 1988. Australasian Chalcidoidea (Hymenoptera) A Biosystematic Revision of Genera of Fourteen Families, with a Reclassification of Species. CAB International, Wallingfort, Oxon, UK. 832 pp.

Cebeci, H. H., G. Grabenweger \& H. Ayberk, 2011. Eulophid parasitoids (Hymenoptera: Eulophidae) of the horse chestnut leafminer, Cameraria ohridella (Lepidoptera: Gracillariidae), from İstanbul, Turkey. Turkish Journal of Zoology, 35 (5): 777-780.

Çıkman, E. \& M. Doğanlar, 2006. Parasitoids of natural populations of Phytomyza orobanchia (Kaltenbach, 1864) (Diptera: Agromyzidae) in southeastern Anatolia. Journal of Applied Science Research, 2 (6): 327-330.

Doğanlar, M., 1982. Hymenopter parasites of some lepidopterous pests in eastern Anatolia. Türkiye Bitki Koruma Dergisi, 6: 197-205.

Doğanlar, M., 1992a. Notes on the species of the nominal subgenus Aprostocetus s.str. of Aprostocetus Westwood, 1833, from Europe and Turkey (Hymenoptera: Eulophidae: Tetrastichinae). Entomofauna Zeitschrift für Entomologie, 13 (31): 513-521.

Doğanlar, M., 1992b. Notes on the species of some genera of Tetrastichinae in Zoologische Staatssammlung München (Hymenoptera: Eulophidae). Entomofauna Zeitschrift für Entomologie, 13 (31): 523-527.

Doğanlar, M., 1992c. A new species of Aprostocetus Westwood, 1833 from Iran. (Hymenoptera: Eulophidae: Tetrastichinae). Entomofauna Zeitschrift für Entomologie, 13 (31): 528-535.

Doğanlar, M., 1993a. A new genus and a new species of Tetrastichinae (Hymenoptera: Eulophidae) from Ghana. Entomofauna Zeitschrift Für Entomologie, 14 (9): 173-178.

Doğanlar, M., 1993b. Systematic works on Sigmophora Rondani, 1867 (Hymenoptera: Eulophidae: Tetrastichinae). Entomofauna Zeitschrift für Entomologie, 14 (9): 179-186.

Doğanlar, M., 1993c. A new genus of Tetrastichinae (Hymenoptera: Eulophidae) from North America. Entomofauna Zeitschrift Für Entomologie, 14 (9): 187-191.

Doğanlar, M., 1993d. A new species of Tachinobia Boucek, 1977 (Hymenoptea: Eulophidae: Tetrastichinae) from Africa. Entomofauna Zeitschrift Für Entomologie, 14 (9): 192-196.

Doğanlar, M., 1994. „ت̈lkemiz Tetrastichinae (Hymenoptera: Eulophidae) türleri üzerinde bazı tesbitler, 83-91“. Türkiye III. Biyolojik Mücadele Kongre Bildirileri, İzmir.

Doğanlar, O. \& M. Doğanlar, 2008. First record of the eucalyptus seed gall wasp, Quadrastichodella nova Girault, 1922, (Eulophidae: Tetrastichinae) from Turkey. Turkish Journal of Zoology, 32 (4): 457-459.

Doğanlar, M., J. LaSalle, E. Sertkaya \& O. Doğanlar, 2009. A new species of Quadrastichus Girault, 1913 (Hymenoptera: Eulophidae: Tetrastichinae) from Hatay, Turkey. Turkish Journal of Zoology, 33 (3): 309-314.

Doğanlar, M., 2011. Parasitoids complex of the olive leaf gall midges, Dasineura oleae (Angelini, 1831) and Lasioptera oleicola Skuhravá, 2011 (Diptera: Cecidomyiidae) in Hatay Turkey, with descriptions of new genus and species from Tetrastichinae (Hymenoptera: Eulophidae). Türkiye Entomoloji Dergisi, 35 (2): 245-264.

Domenichini, G., 1966. Hymenoptera: Eulophidae. Palaearctic Tetrastichinae. Index of Entomophagous insects, 1: 23. Le François, Paris.

Gençer, L. \& M. Doğanlar, 1995. Sivas-Merkez'de Tetrastichinae (Hymenoptera: Eulophidae) üzerine faunistik bir araştırma. Cumhuriyet Üniversitesi Fen- Edebiyat Fakültesi Fen Bilimleri Dergisi, 18: 32-42.

Gençer, L., 2004. Contribution to the knowledge of the family Eulophidae (Hymenoptera: Chalcidoidea) from Turkey, with some new records. Phytoparasitica, 32 (3): 264-273.

Gençer, L., 2009. A new species of Anaprostocetus Graham 1987 (Hymenoptera: Eulophidae) from Turkey. Entomologica Fennica 20 (4) Helsinki: Entomological Society of Finland, 225-227.

Graham, M. W. R. de V., 1987. A Reclassification of the European Tetrastichinae (Hymenoptera; Eulophidae), with a Revision of Certain Genera. Bulletin of the British Museum (Natural History) Entomology series, 55: 1-392.

Graham, M. W. R. de V., 1991. A Reclassification of the European Tetrastichinae (Hymenoptera; Eulophidae), Revision of the Remaining Genera. Memoirs of the American Entomological Institute, 49: 1-322.

Güler, Y. \& M. Kodan, 2010. A new record of ectoparasitoid Melittobia acasta (Walker, 1839) (Hymenoptera: Eulophidae) from Turkey. Mellıfera, HARUM, 10 (1): 34-36. 
Gülperçin, N. \& S. Tezcan, 2010. Türkiye Elateridae (Coleoptera) faunasının endemizm yönünden değerlendirilmesi. BIBAD (Biyoloji Bilimleri Araştırma Dergisi), 3 (2): 111-113.

Karaca, I., G. Japoshvili \& O. Demirözer, 2003. The chalcid parasitoid complex (Hymenoptera: Chalcidoidea) associated with the globose scale (Sphaerolecanium prunasttri Fonscolombe) (Hemiptera: Coccoidea) in Isparta Province, Turkey and some European countries. Zeitschrift für Pflanzenkrankheiten und Pflanzenschutz, 110 (5): 505-511.

Kaya, M. \& B. Kovancı, 2001. Investigations on the biology of Coroebus rubi (L.) (Coleoptera: Buprestidae) in Bursa province of Turkey. Türkiye Entomoloji Dergisi, 25 (4): 275-285.

Koçak, E. \& M. Özdemir, 2012. Parasitic Hymenoptera reared from the insects on Heracleum platytaenium Boiss. (Apiaceae) with new faunistic and biological records. Turkish Journal of Zoology, 36 (2): 201-208.

LaSalle, J., 1994. North American Genera of Tetrastichinae (Hymenoptera: Eulophidea). Journal of Natural History, 28 (1): 109-236.

LaSalle, J., 1999. Ekonomik önemi olan Hymenopterler. Biyoloji ve taksonomi kurs notları. Cumhuriyet Üniversitesi, Fen-Edebiyat Fakültesi Biyoloji Bölümü, Sivas.

Noyes, J. S., 2014. Universal Chalcidoidea database. (Web page: http//www.nhm.ac.uk/entomology/chalcidoids/ index.html) (Date accessed: 26th May 2014).

OILB, 1971. Liste d'identification des entomophages, 27 OILB, Genève $8 \mathrm{pp}$.

Öncüer, C., 1991. A catalogue of the Parasites and Predators of Insect Pests of Turkey. Publication of University of Ege, Faculty of Agriculture No: 505, İzmir, 354 pp.

Özsemerci, F. \& T. Akşit, 2003. Investigations on some biological characteristics and populations fluctuation of Ceroplastes rusci L. (Homoptera: Coccidae) harmful to fig trees in Aydın province. Türkiye Entomoloji Dergisi, 27 (1): 13-25.

Prinsloo, G. L., 1984. An Illustrated Guide to the Parasitic Wasps Associated with Citrus Pests in the Republic of South Africa. Science Bulletin, Department of Agriculture, Republic of South Africa, 402.

Reina, P. \& J. LaSalle, 2003. Key to the world genera of Eulophidae parasitoids (Hymenoptera) of leafmining Agromyzidae (Diptera). (Web page: http://www.ento.csiro.au/science/eulophid_key/Eulophidae/eulophids /html/Aprostocetus.html) (Date accessed: 13th December 2014).

Sakaltaş, E. \& L. Gençer, 2005. Contribution to the knowledge of the Tetrastichinae (Hymenoptera: Eulophidae) from Ankara, Turkey, with some new records. Acta Phytopathologica et Entomologica Hungarica, 40 (3-4): 383-390.

Sakaltaş, E., 2014. Kırıkkale, Çankırı Tetrastichinae (Hymenoptera: Eulophidae) Türleri. Ankara Üniversitesi, Fen Bilimleri Enstitüsü, (Basılmamış) Doktora tezi, Çankaya, Ankara, $123 \mathrm{~s}$.

Ülgentürk, S., 2001. Parasitoids and predators of Coccidae (Homoptera: Coccoidea) species on ornamental plants in Ankara, Turkey. Acta Phytopathologica et Entomologica Hungarica, 36 (3/4): 369-375.

Ülgentürk, S. \& S. Toros, 2001. Natural enemies of the oak scale insect, Eulecanium ciliatum (Douglas) (Hemiptera: Coccidae) in Turkey. Entomologica, Bari 33: 219-224.

Viggiani, G. \& R. Monaco, 2014. Description of a new gall-inducing species of Aprostocetus (Hymenoptera: Eulophidae) on Melilotus indicus from southern Italy. Journal of Entomological and Acarological Research, 46 (1782): 27-29.

Yefremova, Z. A. \& E. N. Yegorenkova, 2005. Two new species of the genus Aprostocetus Westwood from Ul'yanovsk Province of Russia (Hymenoptera: Eulophidae: Tetrastichinae). Zoosystematica Rossica, 14 (1): 155-160.

Yegorenkova, E. N., Z. A. Yefremova \& V. V. Kostjukov, 2007. Contributions to the knowledge of tetrastichine wasps (Hymenoptera: Eulophidae: Tetrastichinae) of the middle Volga region. Entomologicheskoe Obozrenie, 86 (4): 781-796.

Yegorenkova, E. N. \& Z. A. Yefremova, 2011. Baryscapus babiyi Doğanlar, 1993 (Hymenoptera: Eulophidae) is parasitoid of Lasiosina devitata (Diptera: Chloropidae) in Turkey: description of male and discovery of host. Zoosystematica Rossica, 20 (2): 325-329. 